\title{
Comparative Biomechanical Study of Self-tapping and Non Self-tapping Tapered Dental Implants in Artificially Simulated Quality 2 Bone
}

\author{
Yeon-Wha Baek , Duck-Rae Kim, Ju-Hee Park', Young-Jun Lim
}

1. Department of Prosthodontics and Dental Research Institute, School of Dentistry, Seoul National University, Seoul, Korea

\author{
Corresponding Author \\ Young-Jun Lim, DDS, MSD, PhD \\ Department of Prosthodontics, School of Dentistry, Seoul National University, 28 Yeongeon-dong, \\ Jongno-gu, Seoul 110-749, Korea \\ TEL : +82-2-2072-2940 FAX : +82-2-2072-3860 E-mail: limdds@snu.ac.kr
}

Received for publication August 25, 2011; Returned after revision December 2, 2011;

Accepted for publication December 9, 2011

\section{- Abstract}

Purpose: Modifications of implant design have been related to improving initial stability. The purpose of this study was to investigate their respective effect on initial stability between two tapered implant systems (selftapping vs. non-self-tapping) in medium density bone using three different analytic methods.

Materials and Methods: Self-tapping implant (GS III ${ }^{\circledR}$; Osstem Implant Co., Busan, Korea) and non-selftapping implant (Replace Select ${ }^{\circledR}$; Nobel Biocare, Göteborg, Sweden) were investigated. In Solid rigid polyurethane blocks of artificially simulated Quality 2 bone, each of the 5 implants was inserted according to the manufacturer's instructions for medium-bone drilling protocol. Evaluation of initial stability was carried out by recording the maximum insertion torque (IT) and performing the resonance frequency analysis (RFA), and the pull-out test.

Results: The IT and RFA values of self-tapping implant were significantly higher than those of non selftapping implant ( $\mathrm{P}=.009$ and $\mathrm{P}=.047$, respectively). In the pull-out values, no significant differences were found in implants between two groups $(\mathrm{P}=.117)$. Within each implant system, no statistically significant correlation was found among three different outcome variables.

Conclusions: These findings suggest that design characteristics of implant geometry significantly influence the initial stability in medium bone density.

- Key word : Dental implants, Initial stability, Insertion torque, Resonance frequency analysis, Pull-out test

- J Kor Dent Sci. 2011; 4(2) : 52 - 58

() This is an open access article distributed under the terms of the Creative Commons Attribution Non-Commercial License (http:// creativecommons.org/licenses/by-nc/3.0) which permits unrestricted non-commercial use, distribution, and reproduction in any medium, provided the original work is properly cited. 


\section{Introduction}

One of the important factors affecting implant osseointegration is the initial stability of the implants. Securing the initial stability of the implant when placed facilitates the process of tissue healing and osseointegration by restricting the micromotion between the implant and bone surface. Such initial stability also becomes an important scale in determining the loading time of prosthesis ${ }^{1,2)}$. The initial stability of implants is largely affected by three elements: bone substance, surgical technique, and implant design ${ }^{3)}$. Bone substance among those elements has the largest effect on initial stability, and it is not controlled by a clinician. The rate of osseointegration decreases in bone with low density or low ratio of cortical bone, which results in the high rate of implant failure. Low initial stability is said to be the reason.

The taper-type implants developed to increase the implant success rate in bone with poor bone substance can apply lateral compression on the cortical bone of the bone with low density and consequently have uniform force distribution and high value of placement torque compared to cylinder-type implants. Such taper-type implants are also useful when there are anatomic constraints, including ridges with concavities or narrow ridges. Cylindrical wide-bodied implants tend to run the risk of labial perforation due to buccal concavities, while the decrease in diameter toward the apical region of the tapered implant accommodates for the labial concavity ${ }^{4}$. Additionally, the vertical space necessary for placement decreases because the taper-type implants begin to rotate after being inserted into a certain depth of the preformed hole ${ }^{4,5)}$. The self-tapping implants another design for bone with poor substance - were introduced based on the concept wherein it can increase initial stability by applying compression on the neighboring bone without the pre-tapping stage ${ }^{6}$. Such self-cutting implants can also reduce the operation time and simplify the operation process by skipping the tapping process. Moreover, we can avoid the situation wherein wider tapping work than the implant's diameter should be performed because a hand piece cannot be located parallel to the placement angle when the tapping process is clinically performed ${ }^{7}$. Existing studies on the effect of the self-tapping type on the initial stability of the implants showed conflicting results according to the test method and implant fixture type. O'Sullivan et al. ${ }^{8)}$ reported that self-tapping implants showed higher initial stability than non-tapping implants, and that such a result appeared more remarkably in Quality 4 bone with low density. Meanwhile, Rable et al. ${ }^{5}$, Kim et al. ${ }^{6}$, and Kim and $\mathrm{Lim}^{7}$ reported that non-self-tapping implants showed higher initial stability than self-tapping implants.

Many methods of evaluating the initial stability of the implants have been introduced, but no standard was clearly established. Periotest is a device among the non-invasive methods to measure implant stability and was originally designed to measure natural tooth mobility. Nonetheless, many weak points were cited, such as lack of resolution and non-reflection of the minimal change between the boneimplant interface ${ }^{6}$. RFA is a helpful method in measuring stability and osseointegration at the various stages as well as initial stability ${ }^{8}$. The values of resonance frequency are used by changing the range of $3,500 \sim 8,500 \mathrm{~Hz}$ to ISQ (implant stability quotient) $1 \sim 100$. When there is lack of scientific ground in interpreting ISQ, there is no numerical value that is clearly defined as a standard of initial stability, but ISQ of over 65 is interpreted to mean success, whereas ISQ of below 50 means potential failure or increased failure risk. The more popular method of estimating initial stability is the method of measuring the insertion torque (IT). According to a report, such IT measuring method is more objective than RFA or Periotest in measuring bone mineral density or initial stability after the surgery ${ }^{9,10)}$. In case of low IT value, the risk of implant failure increases due to low initial stability, whereas an excessively high IT value can cause implant failure due to the necrosis of neighboring bone. There are also other methods of measuring the strength of the implant-bone interface, such as push-in, pullout, and removal torque test. As invasive methods, however, these are not practically used in dental clinics because they apply nonphysiological force on the implants; they are mainly used for animal testing and in vitro testing ${ }^{7,9,11)}$.

Even though tapered implants were developed for use for immediate placement after tooth extraction and placement in a region with poor bone substance, tapered implants have been generally used clinically in various conditions regardless of bone condition and placement time. There are still few studies on the influence of the self-cutting blade - one of the differences in implant geometry - and of the difference in the implant placement protocol of the relevant implants on the initial stability of the implants.

This study sought to investigate the effect of self-tapping design on the initial stability of implants in medium-density 
bone by selecting two kinds of tapered implants produced by different manufacturers and with features of self-tapping and non self-tapping and by estimating the placement torque, resonance frequency, and pull-out force value after placing the two tapered implants in artificially simulated Quality 2 bone.

\section{Materials and Methods}

Two kinds of tapered implant fixtures were used for this experiment. The implant fixture with diameter of $4.0 \mathrm{~mm}$ and length of $10.00 \mathrm{~mm}$ from GS III system (Osstem Implant Co., Busan, Korea) was used as a self-tapping implant fixture, with the implant fixture having diameter of $4.3 \mathrm{~mm}$ and length of $10.0 \mathrm{~mm}$ from the Replace Select system (Nobel Biocare, Göteborg, Sweden) used as a non self-tapping implant fixture (Fig. 1A).

Solid rigid polyurethane blocks of artificially simulated bone (Sawbones Pacific Research Laboratories Inc., Washington, DC, USA) were used to place such implant fixtures, and two grades by density were utilized to simulate bone equivalent to Quality 2 composed of thick cortical bone and dense cancellous bone based on the bone density classification by Lekhorm \& Zarb ${ }^{12)}$ (Grade \#40 for cortical bone; $1.5 \mathrm{~mm}$ thickness, $0.64 \mathrm{~g} / \mathrm{cc}$ and Grade \#20 for cancellous bone; $28.5 \mathrm{~mm}$ thickness, $0.32 \mathrm{~g} / \mathrm{cc}$ ) (Fig. 1B).

The implants were placed using a CAD/CAM type implant fixture placement device (Osstem Implant Co., Busan, Korea), which is designed to place the implant fixture to a

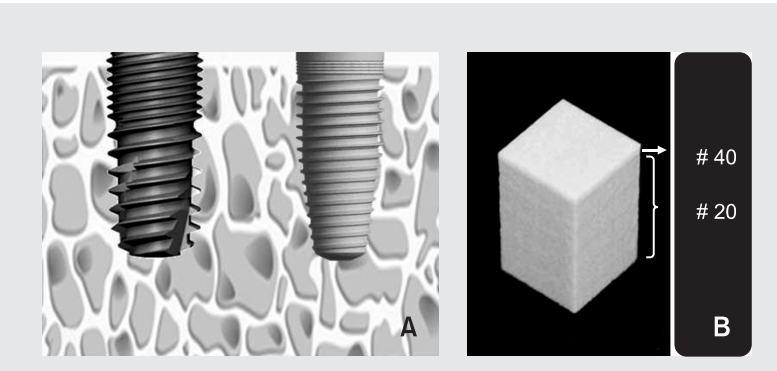

Figure 1. A schematic diagram used in this study. (A) (Left) GS III System designed with cutting edge for self tapping (OSSTEM Implant Co., Busan, Korea), (A) (Right) Replace Select System designed without cutting edge (Nobel Biocare, Gőteborg, Sweden). (B) Quality 2 bone model using Polyurethane synthetic bone blocks.

Yeon-Wha Baek, et al: Comparative Biomechanical Study of Self-tapping and Non SelfTapping Tapered Dental Implants in Artificially Simulated Quality 2 Bone. J Kor Dent Sci 2011. certain depth and at a certain speed through computer control and is able to measure insertion torque simultaneously with implant fixture placement. A total of 5 implants per system were inserted according to the manufacturer's recommended standard for placement in medium density bone (Fig. 2), and the insertion torque value was recorded at the same time.

RFA was performed after placement using OsstellTM Mentor (Integration Diagnostics AB, Göteborg, Sweden); the mean value was calculated through one-time measurement on the buccal, lingual, mesial, and distal sides per fixture after the connection of \#6 (for GS III) and \#13 (for Replace select) SmartPeg ${ }^{\mathrm{TM}}$ (Integration Diagnostics AB, Göteborg, Sweden) to the implant fixtures by hand tightening. A pull-out test was conducted using the Instron Dynamic Material Testing Machine (Instron, Bucks, UK). The pull-out value was measured as a peak in the forcedisplacement curve obtained through the continuous upward pull-out force acting at a speed of $1 \mathrm{~mm} / \mathrm{min}$ after the connection of 2-piece transfer coping with its guide pin linked on the inserted implant to the measuring equipment.
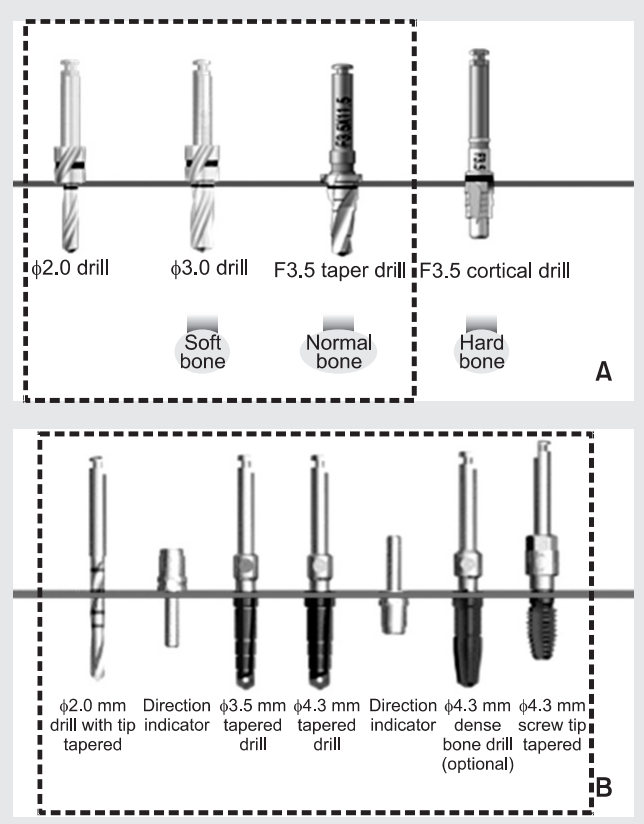

Figure 2. Surgical procedures were performed following procedure recommended by each manufacturer for normal bone. (A) GS III system. (B) Replace select system.

Yeon-Wha Baek, et al: Comparative Biomechanical Study of Self-tapping and Non SelfTapping Tapered Dental Implants in Artificially Simulated Quality 2 Bone. J Kor Dent Sci 2011. 
Statistical analysis was performed using SPSS ver. 12.0 (SPSS Inc., Chicago, IL, USA). To verify the statistical significance of the difference between the two groups based on the results of insertion torque, resonance frequency, and pull-out test, Mann-Whitney test was conducted. To measure the correlation among insertion torque, resonance frequency, and pull-out test, correlation analysis was performed using the Spearman correlation coefficient. Statistical significance was set at $95 \%$ confidence interval.

\section{Results}

The results of the three analytic measurements and statistical analysis are summarized in Table 1 . As a result of measurements, the IT showed no increase at the initial stage of placement, and then rapidly increased up to the peak when placed in the final insertion depth of $10 \mathrm{~mm}$. In the selftapping type GS III System, IT began to increase when

Table 1. Means and standard deviations of three analytic methods

\begin{tabular}{|c|c|c|c|c|}
\hline Assessment method & N & $\begin{array}{c}\text { GS III } \\
\text { MearitsSE }\end{array}$ & $\begin{array}{c}\text { Replace select } \\
\text { MeanISSD }\end{array}$ & $P$ value \\
\hline Insertion torque $(\mathrm{Ncm})$ & 5 & $29.15 \pm 0.83$ & $26.84 \pm 0.75$ & $P<0.01$ \\
\hline RFA (ISQ) & 5 & $71.44 \pm 1.53$ & $68.66 \pm 1.17$ & $P<0.05$ \\
\hline Pull-out torque (N) & 5 & $288.07 \pm 13.69$ & $265.56 \pm 18.65$ & NS. $P=0.117$ \\
\hline
\end{tabular}

RFA: resonance frequency analysis, SD: standard deviation, NS: no significance, N: number of samples.

Yeon-Wha Baek, et al: Comparative Biomechanical Study of Self-tapping and Non SelfTapping Tapered Dental Implants in Artificially Simulated Quality 2 Bone. J Kor Dent Sci 2011.

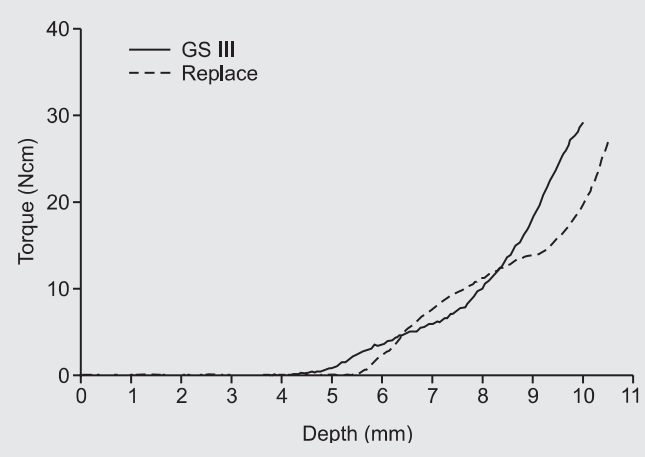

Figure 3. Insertion Torque curve of two implant systems. Significant differences were found between GSIII and Replace. Mann-Whitney test $(\mathrm{P}<0.01)$. $\mathrm{N}=5$.

Yeon-Wha Baek, et al: Comparative Biomechanical Study of Self-tapping and Non SelfTapping Tapered Dental Implants in Artificially Simulated Quality 2 Bone. J Kor Dent Sci 2011. placed to more than the average depth of $4.15 \mathrm{~mm}$ and showed max. 29.1 $\pm 0.83 \mathrm{Ncm}$. For the nonself- tapping type Replace Select System, IT began to increase when placed to more than an average depth of $5.45 \mathrm{~mm}$ and showed max. $26.8 \pm 0.75 \mathrm{Ncm}$. The self-tapping type had higher mean value than the non self-tapping type, and the difference was statistically significant $(\mathrm{P}<0.01)$. The self-tapping-type GS III System also had higher resonance frequency with $71.4 \pm$ 1.54 than the non self-tapping type Replace Select System's $68.7 \pm 1.17$, and the difference was statistically significant

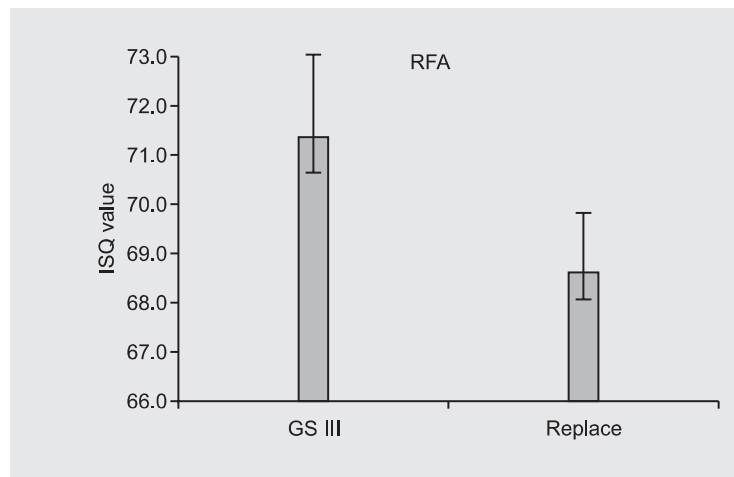

Figure 4. Graph of the ISQ values grouped by implant system. Significant differences were found between GSIII and Replace. Mann-Whitney test $(P<0.01) . \quad N=5$. ISQ: implant stability quotient, RFA: resonance frequency analysis.

Yeon-Wha Baek, et al: Comparative Biomechanical Study of Self-tapping and Non SelfTapping Tapered Dental Implants in Artificially Simulated Quality 2 Bone. J Kor Dent Sci 2011.

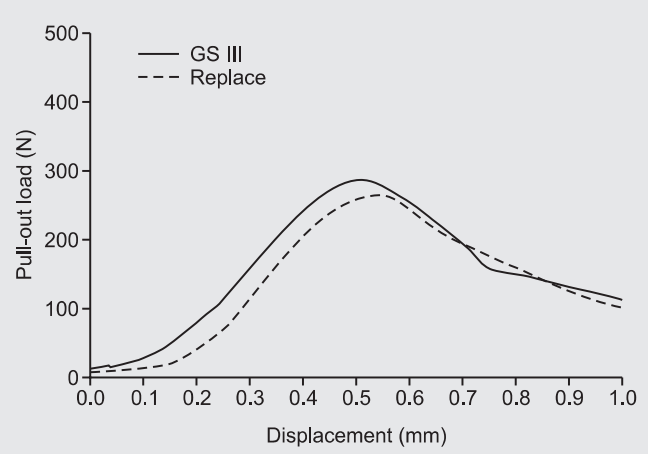

Figure 5. Load-displacement curves obtained by pull-out test, yielding the breakage strength of bone-titanium integration as a pull-out value. Significant differences were found between GSIII and Replace. Mann-Whitney test $(\mathrm{P}<0.01)$. $\mathrm{N}=5$.

Yeon-Wha Baek, et al: Comparative Biomechanical Study of Self-tapping and Non SelfTapping Tapered Dental Implants in Artificially Simulated Quality 2 Bone. J Kor Dent Sci 2011. 
$(\mathrm{P}<0.05)$. The pull-out force showed an aspect of decline after increasing up to a certain level. The self-tapping type GS III System showed higher maximum value with 288.07 $\pm 13.69 \mathrm{~N}$ than the non self-tapping type Replace Select System's $265.56 \pm 18.65 \mathrm{~N}$, but the difference was not statistically significant $(\mathrm{P}=0.117)$ (Table 1 , Fig. $3 \sim 5)$. As a result of analyzing the correlation among insertion torque, resonance frequency, and pull-out force, the difference in measured value was found to have no statistical significance.

\section{Discussion}

This experiment compared the initial stability between two kinds of tapered implants through IT, RFA, and pull-out test among the various evaluation methods of initial stability. In medium density bone, the IT value and ISQ of the selftapping type GS III were higher than the non self-tapping type Replace Select $(\mathrm{P}<0.01, \mathrm{P}<0.05$ respectively), and the difference was statistically significant. The pull-out value of GS III was also higher than Replace Select, but the difference was not statistically significant $(\mathrm{P}=0.117)$.

The higher IT value of GS III than Replace Select can be attributed to the development background and principle of the self-tapping implant. In other words, GS III can do away with the tapping process in Quality 2 bone during operation due to the self-tapping blade designed in a fixture, whereas Replace Select requires carrying out the process of tapping as much as the same diameter of a fixture during placement in Quality 2 bone (Fig. 2). Therefore, the GS III implant fixture with no tapping can be said to show high IT value in applying lateral pressure to the surrounding bone. Originally developed to improve the initial stability of the implants in low-density bone, the self-tapping implant offers convenience in operation since the tapping process can be done away with; such convenience leads to an attempt to use the self-tapping implant in medium-density bone. According to a report, the self-tapping implant in tapered form may give rise to the case wherein clinical placement in the bone with over medium density level is impossible or cause implant failure including osteonecrosis due to excessive IT. ${ }^{13)}$ The study by Sakoh et al. ${ }^{14)}$ reported that, in case the tapping process is skipped according to the original drilling protocol in the displacement test for the taper-type, self-cutting implants, all implants could be placed in the maxillary bone without any additional process to as much depth as required; in mandibular bone, however, 11 out of the 62 implants placed in Quality 2 bone could be placed only by using a hand-drilling driver to as much depth as required, and 2 implants could not be placed in Quality 1 bone. As a result of placement in simulated Quality 2 bone in this test, all implants could be placed without any resistance and to as much depth as required, and excessive IT did not occur in the two kinds of implants. Proper ISQ value could also be obtained.

RFA considers the implant and surrounding bone as one unit and recognizes stiffness change as the change between the implant and the bone. The following are the influence factors: (1) stiffness of the implant itself, which is influenced by the implant's length, diameter, shape, and material; (2) stiffness of the implant-bone interface, which is determined by the combination of implant and bone, and; (3) stiffness of the surrounding tissue, which is influenced by the composition ratio and bone density of cortical bone and cancellous bone ${ }^{9)}$.

Roze et al. ${ }^{15)}$ reported that the value of resonance frequency had no relationship with the histomorphological factor of cancellous bone but was related to the thickness of cortical bone only. According to Friberg et al. ${ }^{16)}$ insertion torque and resonance frequency showed high correlation when placed in up to one-third of the region of cortical bone ( $\mathrm{r}=0.84$ ), and resonance frequency was determined by the thickness and density of cortical bone. Another study reported that the height and bone substance of the implant and transducer exposed to the upper part of the bone had substantial influence on the RFA value. ${ }^{17-20)}$ In this study, the length of the implant and transducer exposed to the upper part of bone was constantly maintained, and the influence of the variables on RFA was minimized by constantly setting bone substance as Quality 2 bone density. As a result, the ISQ value of the self-tapping implant was found to be significantly higher than that of the non self-tapping implant; this was consistent with the result of the test by O'Sullivan, et al, i.e., the ISQ value of the tapered self-tapping implant was significantly higher than that of the standard non-self tapping implant in Quality 4 bone ${ }^{8)}$.

Meanwhile, there was also a study wherein the resonance frequency value was not a standard to compare the initial stability among different implant systems. Ersanli et al. ${ }^{19}$ maintained that RFA was useful in measuring the change of implant stability from the long-term perspective but was not an appropriate method for comparing different implant 
systems at a certain stage. The pull-out value is judged as resistance to the initial force, and the one wherein the small implant fixture's displacement responds to the initial force acting after placement means the product has good initial stability. In this test, the value showed rapid decline again after a linear increase up to the point wherein the mechanical combination of bone and implant was destroyed. The maximum mean value of the self-tapping type GS III System was higher than the non-self-tapping type Replace Select System, but the difference was not statistically significant.

Among studies comparing the self-tapping and non-self tapping implants, there were studies reporting that the initial stability of the non self-tapping implant was excellent, unlike the result of this test ${ }^{4-7)}$. In an experiment similar to the one in this study on Quality 4 bone, the result of implant placement without the tapping stage in both self-tapping and non self-tapping implants according to the drilling protocol revealed higher initial stability in the non selftapping implant. Likewise, the result of placing the same type of implant fixture in Quality 2 bone except a selftapping blade according to the drilling protocol revealed higher initial stability in the non self-tapping implant. In the case of the same operation process for the placement of self tapping and non self-tapping implants, the initial stability of the self-tapping implant can be estimated to decrease due to the reduction of thread area and consequent decrease of contact area with the surrounding one.

In most existing studies on the influence of self-tapping design on the initial stability of the implants, there were limitations, i.e., the self-tapping implant was not the only variable due to the non-unification of the implant shapes between the experimental group and the control group, there was a difference in the drilling protocol by manufacturer, and the influence of the self-tapping design on initial stability could not be identified due to the test under conditions wherein bone density was not constantly maintained. A simulated polyurethane bone block is put to good use for test and training in orthopedics, with a recent experimental study reporting that simulated bone in the market is more homogeneous and consistent than the real cancellous bone ${ }^{21)}$, that such homogenous bone substance could reduce the number of experiments necessary to obtain statistical significance, and that the standard deviation could be reduced to one-fourth of that in the same test using real bone $^{22)}$. In this test, more homogenous Quality 2 bone density was reproduced using simulated bone; the other variables except implant design were minimized by using a device that could continuously apply constant magnitude of force to exclude the influence of hand pressure during the measurement of insert torque and pull-out torque. Since the self-tapping implant and the non self-tapping implant came from different manufacturers and had differences in the diameter of the implant and number, shape, and width of the thread, however, the limitations of this study were the tapping blade acting as a variable and the insufficient sample number.

\section{Conclusion}

Within the limitations of this study, the following conclusions were drawn:

1. When implant placement in medium-density bone was performed according to the normal bone operation protocol of the manufacturer, the self-tapping type showed higher values for insertion torque and resonance frequency than those of the non self-tapping type.

2. The values of pull-out torque in the two types showed no significant difference.

3 . The correlation among insertion torque, maximum pullout torque, and resonance frequency per group had no statistical significance.

The result of this test revealed that the initial stability of the self-tapping type implant without the tapping process in medium-density bone was higher than that of the non-self tapping type implant. It is important for the clinician to acknowledge that the critical factor contributing to initial stability is the difference of implant drilling protocol according to the their own design characteristics. 
1. Friberg B, Jemt T, Lekholm U. Early failures in 4641 consecutively placed Branemark dental implants. A study from stage I surgery to the connection of completed prostheses. Int J Oral Maxillofac Implants. 1991; 6: 142-6.

2. Ivanoff CJ, Sennerby L, Lekholm U. Influence of mono-and bicortical anchorage on the integration of titanium implants. A study in the rabbit tibia. Int J Oral Maxillofac Surg. 1996; 25: 229-35.

3. Meredith N. Assessment of implant stability as a prognostic determinant. Int J Prosthodont. 1998; 11: 491-501.

4. Chong L, Khocht A, Suzuki JB, Gaughan J. Effect of implant design on initial stability of tapered implants. J Oral Implantol. 2009; 35: 130-5.

5. Rabel A, Köhler SG, Schmidt-Westhausen AM. Clinical study on the primary stability of two dental implant systems with resonance frequency analysis. Clin Oral Investig. 2007; 11: 257-65.

6. Kim DR, Kim MJ, Kwon HB, Lee SH, Lim YJ. In vitro study on the initial stability of two tapered dental implant systems in poor bone quality. J Korean Acad Stomato Func Occl. 2009; 25: 389-99.

7. Kim YS, Lim YJ. Primary stability and self-tapping blades: biomechanical assessment of dental implants in medium-density bone. Clin Oral Implants Res. 2011; 22: 1179-84.

8. O'Sullivan D, Sennerby L, Meredith N. Measurements comparing the initial stability of five designs of dental implants: a human cadaver study. Clin Implant Dent Relat Res. 2000; 2: 85-92.

9. Atsumi M, Park SH, Wang HL. Methods used to assess implant stability: current status. Int J Oral Maxillofac Implants. 2007; 22: 74354.

10. Friberg B, Sennerby L, Roos J, Johansson P, Strid CG, Lekholm U. Evaluation of bone density using cutting resistance measurements and microradiography: an in vitro study in pig ribs. Clin Oral Implants Res. 1995; 6: 164-71.

11. Homolka P, Beer A, Birkfellner W, Nowotny R, Gahleitner A, Tschabitscher M, Bergmann H. Bone mineral density measurement with dental quantitative $\mathrm{CT}$ prior to dental implant placement in cadaver mandibles: pilot study. Radiol. 2002; 224: 247-52.

12. Brånemark P-I, Lekholm U, Zarb GA, Albrektsson T: Tissue-Integrated
Prostheses. 1st ed. Chicago: Quintessence Publishing Co.; 1985: p.202.

13. Niimi A, Ozeki K, Ueda M, Nakayama B. A comparative study of removal torque of endosseous implants in the fibula, iliac crest and scapula of cadavers: preliminary report. Clin Oral Implants Res. 1997; 8: 286-9.

14. Sakoh J, Wahlmann U, Stender E, Nat R, Al-Nawas B, Wagner W. Primary stability of a conical implant and a hybrid, cylindric screw-type implant in vitro. Int J Oral Maxillofac Implants. 2006; 21: 560-6.

15. Roze J, Babu S, Saffarzadeh A, Gayet-Delacroix M, Hoormaert A, Layrolle P. Correlating implant stability to bone structure. Clin Oral Implants Res. 2009; 20: 1140-5.

16. Friberg B, Gröndahl K, Lekholm U. A new self-tapping Branemark implant: clinical and radiographic evaluation. Int J Oral Maxillofac Implants. 1992; 7: 80-5.

17. Akca K, Akkocaoglu M, Comert A, Tekdemir I, Cehreli MC. Human ex vivo bone tissue strains around immediately loaded implants supporting maxillary overdentures. Clin Oral Implants Res. 2005; 16: 715-22.

18. Akkocaoglu M, Uysal S, Tekdemir I, Akca K, Cehreli MC. Implant design and intraosseous stability of immediately placed implants: a human cadaver study. Clin Oral Implants Res. 2005; 16: 202-9.

19. Ersanli S, Karabuda C, Beck F, Leblebicioglu B. Resonance frequency analysis of one-stage dental implant stability during the osseointegration period. J Periodontol. 2005; 76: 1066-71.

20. Meredith N, Book K, Friberg B, Jemt T, Sennerby L. Resonance frequency measurements of implant stability in vivo. A cross-sectional and longitudinal study of resonance frequency measurements on implants in the edentulous and partially dentate maxilla. Clin Oral Implants Res. 1997; 8: 226-33.

21. Calvert KL, Trumble KP, Webster TJ, Kirkpatrick LA. Characterization of commercial rigid polyurethane foams used as bone analogs for implant testing. J Mater Sci Mater Med. 2010; 21: 1453-61.

22. Tabassum A, Meijer GJ, Wolke JGC, Jansen JA. Influence of the surgical technique and surface roughness on the primary stability of an implant in artificial bone with a density equivalent to maxillary bone: a laboratory study. Clin Oral Implants Res. 2009; 20: 327-32. 